\title{
Entire solutions of a class of algebraic Briot-Bouquet differential equations utilizing majority concept
}

\author{
Rabha W. Ibrahim ${ }^{1,2^{*}}$ (D) and Dumitru Baleanu $u^{3,4,5}$
}

\author{
"Correspondence: \\ rabhaibrahim@tdtu.edu.vn \\ 'Informetrics Research Group, Ton \\ Duc Thang University, Ho Chi Minh \\ City, Vietnam \\ ${ }^{2}$ Faculty of Mathematics \& Statistics, \\ Ton Duc Thang University, Ho Chi \\ Minh City, Vietnam \\ Full list of author information is \\ available at the end of the article
}

\begin{abstract}
In this effort, the analytic solution of a class of algebraic Briot-Bouquet differential equations ( $A B B D E$ ) in the open unit disk is investigated by making use of a major theory. The class is presented by the formula

$$
\begin{aligned}
& \alpha_{1} \varphi^{\prime 3}(z)+\alpha_{2} \varphi^{\prime 2}(z) \varphi(z)+\alpha_{3} \varphi^{\prime}(z) \varphi^{2}(z)+\aleph_{\varphi}^{k}(z)=0 \\
& \aleph_{\varphi}^{k}(z):=a_{k} \varphi^{k}(z)+a_{k-1} \varphi^{k-1}(z)+\cdots+a_{1} \varphi(z)+a_{0} .
\end{aligned}
$$

The conformal analysis (angle-preserving) of the ABBDEs is considered. Analytic outcomes of the ABBDEs are indicated by employing the major method. Some special cases are investigated.
\end{abstract}

MSC: $30 C 55 ; 30 C 45$

Keywords: Analytic function; Subordination; Univalent function; Open unit disk; Algebraic differential equations; Majorization method

\section{Introduction}

The algebraic Briot-Bouquet differential equation (ABBDE) is a significant nonlinear differential equation which occurs in many applications such as neuroscience, geometry, economy, banking studies, and physics. The most important property of ABBDE is that it shows that every meromorphic solution of ABBDE belongs to the class of meromorphic functions containing elliptic functions and their degenerates, i.e., the rational functions of one exponential $e^{\zeta z}, \zeta \in \mathbb{C}, z \in \mathbb{U}=\{z \in \mathbb{C}:|z|<1\}$ and rational functions. Many consequences for higher-order $\mathrm{ABBDE}$ are also presented in a sequence of documents (see [1-12]).

The majorization inequality theory (real and complex) has many applications in our real life [13]. For example, they have measured the economy, specifically in the incomedistribution (economic inequality), that is, the distribution of income between the primary factors of production. New economists have, furthermore, addressed issues of income distribution, but have absorbed further on the distribution of income persons and

(c) The Author(s) 2020. This article is licensed under a Creative Commons Attribution 4.0 International License, which permits use, sharing, adaptation, distribution and reproduction in any medium or format, as long as you give appropriate credit to the original author(s) and the source, provide a link to the Creative Commons licence, and indicate if changes were made. The images or other third party material in this article are included in the article's Creative Commons licence, unless indicated otherwise in a credit line to the material. If material is not included in the article's Creative Commons licence and your intended use is not permitted by statutory regulation or exceeds the permitted use, you will need to obtain permission directly from the copyright holder. To view a copy of this licence, visit http://creativecommons.org/licenses/by/4.0/. 
households. Important theoretical and policy concerns consist of the balance between income majorization inequality and economic growth, and their often-inverse association. Moreover, the Gini coefficient measures the inequality among the values of a frequency distribution (for example, levels of income) [14]. A Gini coefficient of zero states perfect equality, where all values are the same (for example, where everyone has the same income) [15]. Therefore, the solution of any class of differential equations majoring by a convex function or by special function is very attractive, especially in wealth and health distribution [16].

In this paper, we investigate a class of ABBDEs in the open unit disk. We study the geometric representation for this class and majorize them by employing special function in $\mathbb{U}$ including the exponential function.

\section{Complex ABBDEs}

A special class of ABBDEs is studied in [7] taking the structure

$$
\alpha_{1} \varphi^{\prime 3}(z)+\alpha_{2} \varphi^{\prime 2}(z) \varphi(z)+\alpha_{3} \varphi^{\prime}(z) \varphi^{2}(z)+\aleph_{\varphi}^{k}(z)=0
$$

where $\alpha_{\imath} \in \mathbb{C}, \imath=1,2,3$, and

$$
\aleph_{\varphi}^{k}(z):=a_{k} \varphi^{k}(z)+a_{k-1} \varphi^{k-1}(z)+\cdots+a_{1} \varphi(z)+a_{0}
$$

where $a_{\jmath}, J=0,1, \ldots, k$, are constants (real or complex).

Now, we reorganize (2.1) and consider the geometric possessions by designing some classes of normalized analytic functions in $\mathbb{U}$. Then the resolution is majorized by engaging special functions in $\mathbb{U}$. Equation (2.1) suggests the homogeneous form when $\alpha_{1} \neq 0$,

$$
\left(\frac{z \varphi^{\prime}(z)}{\varphi(z)}\right)^{2}+b_{2} z\left(\frac{z \varphi^{\prime}(z)}{\varphi(z)}\right)+b_{3} z^{2}=0, \quad z \in \mathbb{U}, b_{2}=\frac{\alpha_{2}}{\alpha_{1}}, b_{3}=\frac{\alpha_{3}}{\alpha_{1}}
$$

To study Eq. (2.2) geometrically, we request the following theory.

Definition 2.1 An analytic function $h$ is subordinated to an analytic function $g$, written $h \prec g$, if substitutes for an analytic function $w$ with $|w(z)| \leq|z|$ such that $h=(g(w))$ (see [17]). The Ma-Minda classes $S^{*}(\rho)$ and $K(\rho)$ of starlike and convex functions respectively indicated by $\left(\frac{z h^{\prime}(z)}{h(z)}\right) \prec \rho(z)$ and $\left(1+\frac{z h^{\prime \prime}(z)}{h^{\prime}(z)}\right) \prec \rho(z)$, where $\rho$ has a positive real part in $\mathbb{U}$, $\rho(0)=1, \rho^{\prime}(0)>1$ and maps $\mathbb{U}$ onto a starlike-domain.

We recognize our study by applying the above inequality to formulate the following special class.

Definition 2.2 A function of normalized expansion $\varphi(z)=z+\sum_{n=2}^{\infty} \varphi_{n} z^{n}, z \in \mathbb{U}$ is said to be in the class $\mathbf{M}(\rho)$ if and only if

$$
\begin{aligned}
& P(z):=\left(\frac{z \varphi^{\prime}(z)}{\varphi(z)}\right)^{2}+b_{2} z\left(\frac{z \varphi^{\prime}(z)}{\varphi(z)}\right)+b_{3} z^{2} \prec \rho(z), \\
& \left(\rho(0)=1, \rho^{\prime}(0)>1, z \in \mathbb{U}\right) .
\end{aligned}
$$


It is clear that $P(0)=1$. For example, let $\varphi(z)=z /(1-z), z \in \mathbb{U}$, then

$$
P(z)=1+\left(b_{2}+2\right) z+\left(b_{2}+b_{3}+3\right) z^{2}+\left(b_{2}+4\right) z^{3}+\left(b_{2}+5\right) z^{4}+\left(b_{2}+6\right) z^{5}+O\left(z^{6}\right) .
$$

Also, for $\varphi(z)=z /(1-z)^{2}, z \in \mathbb{U}$, we have

$$
\begin{aligned}
P(z)= & 1+\left(b_{2}+4\right) z+\left(2 b_{2}+b_{3}+8\right) z^{2}+2\left(b_{2}+6\right) z^{3}+2\left(b_{2}+8\right) z^{4} \\
& +2\left(b_{2}+10\right) z^{5}+O\left(z^{6}\right) .
\end{aligned}
$$

In the sequel, we theorize a starlike function with positive real part such as $e^{z}$ (this function yields an oscillation solution of Eq. (2.1) [7]) and a convex function (univalent)

$$
\rho_{e}(z)=\frac{z}{e^{z}-1}=1-\frac{z}{2}+\frac{z^{2}}{12}-\frac{z^{4}}{720}+\cdots
$$

as well as

$$
\varrho_{e}(z):=1 / \rho_{e}(z)=1+\frac{z}{2}+\frac{z^{2}}{6}+\frac{z^{3}}{24}+\frac{z^{4}}{120}+\cdots
$$

is convex univalent in $\mathbb{U}$ (see [17], p. 415). Note that the coefficients are converging to the Bernoulli numbers. Moreover, the real part of the function $\varrho_{e}(z)=\left(e^{z}-1\right) / z$ satisfies the inequality

$$
\Re\left(\frac{e^{\eta z}-1}{\eta z}\right) \geq \frac{1}{2}, \quad 0<\eta \leq 1.793 \ldots
$$

Hence, $\Re\left(\frac{e^{\eta z}-1}{\eta z}\right) \geq 1 / \rho_{e}(-1)=\frac{1}{2}$.

Our calculation is established by the analytic method of Caratheodory functions which are characterized in [18]. Consequently, by making use of major theory, we approximate $\aleph_{\varphi}^{k}(z)$ by a special type of $\rho(z), z \in \mathbb{U}$ denoted by $\aleph_{\varphi}^{k}(z) \ll \rho(z)$. Note that the two functions are under majorization if and only if $\left|a_{J}\right| \leq\left|\rho_{J}\right|$ for all $J=1,2, \ldots$, where $a_{J}$ and $\rho_{J}$ are the coefficients of $\aleph_{\varphi}^{k}(z)$ and $\rho(z)$ respectively. In this situation, we demonstrate adequate conditions of the coefficient constraints of $\aleph_{\varphi}^{k}(z)$, for altered values of $k=0,1, \ldots$, using a Caratheodory theory.

\section{Computations}

In this position, we demonstrate our theoretical results.

Theorem 3.1 Let the function $\varphi \in M(\rho)$ achieve the inequality

$$
1+\curlyvee\left(\frac{z P^{\prime}(z)}{[P(z)]^{k}}\right) \prec z+\sqrt{z^{2}+1}, \quad k=0,1,2,
$$

where $\curlyvee$ is a constant and $P(z)=\left(\frac{z \varphi^{\prime}(z)}{\varphi(z)}\right)^{2}+b_{2} z\left(\frac{z \varphi^{\prime}(z)}{\varphi(z)}\right)+b_{3} z^{2}$. Then

$$
P(z) \prec \rho_{e}(z)=\frac{z}{e^{z}-1}, \quad z \in \mathbb{U} .
$$

When $\curlyvee \geq \max \curlyvee_{k}$, 


$$
\min \curlyvee_{0}=\frac{-((e-1)(-2+\sqrt{2}+\log (2)-\log (1+\sqrt{2})))}{(e-2)} \approx 1.8516 \ldots
$$

and

$$
\max \curlyvee_{0}=(e-1)(\sqrt{2}+\log (2)+\log (\sqrt{2}-1)) \approx 2.106 \ldots
$$

$$
\min \curlyvee_{1}=\frac{(2-\sqrt{2}-\log (2)+\log (1+\sqrt{2}))}{\log (e-1)} \approx 1.5 \ldots
$$

and

$$
\max \curlyvee_{1}=\frac{(-\sqrt{(} 2)-\log (2)-\log (\sqrt{(}(2)-1))}{(\log (e-1)-1)} \approx 2.839 \ldots
$$

$$
\min \curlyvee_{2}=\frac{2-\sqrt{2}+\log (1 / 2+1 / \sqrt{2})}{(e-2)} \approx 1.077 \ldots
$$

and

$$
\max \curlyvee_{2}=e(\sqrt{2}+\log (2)-\log (1+\sqrt{2})) \approx 3.33 \ldots
$$

Proof Case I: $k=0 \Rightarrow 1+\curlyvee\left(z P^{\prime}(z)\right) \prec z+\sqrt{z^{2}+1}$.

Set a function $\Gamma_{\curlyvee}: \mathbb{U} \rightarrow \mathbb{C}$ admitting the structure

$$
\Gamma_{\curlyvee}(z)=1+\frac{1}{\curlyvee}\left(z+\sqrt{z^{2}+1}-\log \left(1+\sqrt{z^{2}+1}\right)-1+\log (2)\right) .
$$

Clearly, $\Gamma_{\curlyvee}(z)$ is analytic in $\mathbb{U}$ such that $\Gamma_{\curlyvee}(0)=1$ and it is the outcome of the differential equation

$$
1+\curlyvee\left(z \Gamma_{\curlyvee}^{\prime}(z)\right)=z+\sqrt{z^{2}+1}, \quad z \in \mathbb{U} .
$$

Consequently, we have $\mathfrak{Q}(z):=\curlyvee\left(z \Gamma_{\curlyvee}^{\prime}(z)\right)=z+\sqrt{z^{2}+1}-1$ is starlike in $\mathbb{U}$. Then for $\mathfrak{J}(z):=$ $\mathfrak{Q}(z)+1$, we conclude that

$$
\Re\left(\frac{z \mathfrak{Q}^{\prime}(z)}{\mathfrak{Q}(z)}\right)=\mathfrak{R}\left(\frac{z \mathfrak{J}^{\prime}(z)}{\mathfrak{Q}(z)}\right)>0
$$

Thus, the Miller-Mocanu lemma (see [17], p. 132) yields that

$$
1+\curlyvee\left(z P^{\prime}(z)\right) \prec 1+\curlyvee z \Gamma_{\curlyvee}^{\prime}(z) \quad \Rightarrow \quad P(z) \prec \Gamma_{\curlyvee}(z)
$$


To finish this event, we only need to prove that $\Gamma_{\curlyvee}(z) \prec \rho_{e}(z)$. Clearly, the function $\Gamma_{\curlyvee}(z)$ is increasing in the interval $(-1,1)$ that has the inequality

$$
\Gamma_{\curlyvee}(-1) \leq \Gamma_{\curlyvee}(1) .
$$

Because the function $\rho_{e}(z)$ achieves the inequality for real $\vartheta$

$$
\frac{1}{e-1} \leq \mathfrak{R}\left(\rho_{e}(z)\right) \approx 1-\frac{\cos (\vartheta)}{2}+\sum_{n=1}^{\infty} \frac{\beta_{2 n} \cos (2 n \vartheta)}{(2 n) !} \leq \frac{e}{e-1},
$$

then the consequence inequality holds

$$
\frac{1}{e-1} \leq \Gamma_{\curlyvee}(-1) \leq \Gamma_{\curlyvee}(1) \leq \frac{e}{e-1}
$$

if $\curlyvee$ fulfills the upper and lower bounds

$$
\min \curlyvee_{0}=\frac{-((e-1)(-2+\sqrt{2}+\log (2)-\log (1+\sqrt{2})))}{(e-2)} \approx 1.8516 \ldots
$$

and

$$
\max \curlyvee_{0}=(e-1)(\sqrt{2}+\log (2)+\log (\sqrt{2}-1)) \approx 2.106 \ldots
$$

This yields the subordination inequalities

$$
\Gamma_{\curlyvee}(z) \prec \frac{z}{e^{z}-1} \quad \Rightarrow \quad P(z) \prec \frac{z}{e^{z}-1}, \quad z \in \mathbb{U} .
$$

Case II: $k=1 \Rightarrow 1+\curlyvee\left(\frac{z P^{\prime}(z)}{P(z)}\right) \prec z+\sqrt{z^{2}+1}$.

Now we present a function $\Pi_{\curlyvee}: \mathbb{U} \rightarrow \mathbb{C}$ structuring by the formula

$$
\Pi_{\curlyvee}(z)=\exp \left(\frac{1}{\curlyvee}\left(z+\sqrt{z^{2}+1}-\log \left(1+\sqrt{z^{2}+1}\right)-1+\log (2)\right)\right) .
$$

Obviously, $\Pi_{\curlyvee}(z)$ is analytic in $\mathbb{U}$ having $\Pi_{\curlyvee}(0)=1$ and it is an outcome of the differential equation

$$
1+\curlyvee\left(\frac{z \Pi_{\curlyvee}^{\prime}(z)}{\Pi_{\curlyvee}(z)}\right)=z+\sqrt{z^{2}+1}, \quad z \in \mathbb{U} .
$$

By using $\mathfrak{Q}(z)=z+\sqrt{z^{2}+1}-1$, which is starlike in $\mathbb{U}$ and $\mathfrak{J}(z)=\mathfrak{Q}(z)+1$, we get

$$
\Re\left(\frac{z \mathfrak{Q}^{\prime}(z)}{\mathfrak{Q}(z)}\right)=\Re\left(\frac{z \mathfrak{J}^{\prime}(z)}{\mathfrak{Q}(z)}\right)>0, \quad z \in \mathbb{U}
$$

Then again, by Miller-Mocanu lemma, we obtain

$$
1+\curlyvee\left(\frac{z P^{\prime}(z)}{P(z)}\right) \prec 1+\curlyvee\left(\frac{z \Pi_{\curlyvee}^{\prime}(z)}{\Pi_{\curlyvee}(z)}\right) \Rightarrow P(z) \prec \Pi_{\curlyvee}(z) .
$$


Consequently, the following inequality holds:

$$
\frac{1}{e-1} \leq \Pi_{\curlyvee}(-1) \leq \Pi_{\curlyvee}(1) \leq \frac{e}{e-1}
$$

if $\curlyvee$ has the upper and lower bounds

$$
\min \curlyvee_{1}=\frac{(2-\sqrt{2}-\log (2)+\log (1+\sqrt{2}))}{\log (e-1)} \approx 1.5 \ldots
$$

and

$$
\max \curlyvee_{1}=\frac{(-\sqrt{(2)}-\log (2)-\log (\sqrt{(2)}-1))}{(\log (e-1)-1)} \approx 2.839 \ldots
$$

This indicates the subordination inequalities

$$
\Pi_{\curlyvee}(z) \prec \frac{z}{e^{z}-1} \quad \Rightarrow \quad P(z) \prec \frac{z}{e^{z}-1}, \quad z \in \mathbb{U} .
$$

Case III: $k=2 \Rightarrow 1+\curlyvee\left(\frac{z P^{\prime}(z)}{P^{2}(z)}\right) \prec z+\sqrt{z^{2}+1}$.

Now, we introduce a function $\Pi_{\curlyvee}: \mathbb{U} \rightarrow \mathbb{C}$ having the formal

$$
\Theta_{\curlyvee}(z)=\left(1-\frac{1}{\curlyvee}\left(z+\sqrt{z^{2}+1}-\log \left(1+\sqrt{z^{2}+1}\right)-1+\log (2)\right)\right)^{-1} .
$$

Clearly, $\Pi_{\curlyvee}(z)$ is analytic in $\mathbb{U}$ admitting $\Pi_{\curlyvee}(0)=1$, and it is the outcome of the differential equation

$$
1+\curlyvee\left(\frac{z \Theta_{\curlyvee}^{\prime}(z)}{\Theta_{\curlyvee}(z)}\right)=z+\sqrt{z^{2}+1}, \quad z \in \mathbb{U}
$$

By utilizing $\mathfrak{Q}(z)=z+\sqrt{z^{2}+1}-1$, which is starlike in $\mathbb{U}$ and $\mathfrak{J}(z)=\mathfrak{Q}(z)+1$, we get

$$
\Re\left(\frac{z \mathfrak{Q}^{\prime}(z)}{\mathfrak{Q}(z)}\right)=\Re\left(\frac{z \mathfrak{J}^{\prime}(z)}{\mathfrak{O}(z)}\right)>0, \quad z \in \mathbb{U} .
$$

Consequently, by Miller-Mocanu lemma, we get

$$
1+\curlyvee\left(\frac{z P^{\prime}(z)}{P^{2}(z)}\right) \prec 1+\curlyvee\left(\frac{z \Theta_{\curlyvee}^{\prime}(z)}{\Theta_{\curlyvee}^{2}(z)}\right) \Rightarrow P(z) \prec \Theta_{\curlyvee}(z)
$$

Accordingly, we attain

$$
\frac{1}{e-1} \leq \Theta_{\curlyvee}(-1) \leq \Theta_{\curlyvee}(1) \leq \frac{e}{e-1}
$$

if $\curlyvee_{2}$ admits the upper and lower bounds

$$
\min \curlyvee_{2}=\frac{2-\sqrt{2}+\log (1 / 2+1 / \sqrt{2})}{(e-2)} \approx 1.077 \ldots
$$


and

$$
\max \curlyvee_{2}=e(\sqrt{2}+\log (2)-\log (1+\sqrt{2})) \approx 3.33 \ldots
$$

This yields the subordination inequalities

$$
\Theta_{\curlyvee}(z) \prec \frac{z}{e^{z}-1} \quad \Rightarrow \quad P(z) \prec \frac{z}{e^{z}-1}, \quad z \in \mathbb{U} .
$$

The next result studies the subordination with respect to the function

$$
\varrho_{e}(z)=\frac{e^{z}-1}{z}, \quad z \in \mathbb{U}
$$

Theorem 3.2 Let the assumptions of Theorem 3.1 hold for a constant $\curlywedge$ where

$$
1+\curlywedge\left(\frac{z P^{\prime}(z)}{[P(z)]^{k}}\right) \prec z+\sqrt{z^{2}+1}, \quad k=0,1,2 .
$$

Then

$$
P(z) \prec \varrho_{e}(z)=\frac{e^{z}-1}{z}, \quad z \in \mathbb{U} .
$$

When $\curlywedge \geq \max \curlywedge_{k}$,

$$
\min \curlywedge_{0}=\frac{(\sqrt{2}+\log (2)+\log (\sqrt{2}-1))}{(e-2)} \approx 1.706 \ldots
$$

and

$$
\max \curlywedge_{0}=-e(-2+\sqrt{2}+\log (2)-\log (1+\sqrt{(2)})) \approx 2.10399 \ldots
$$

$$
\min \curlywedge_{1}=\frac{(-2+\sqrt{2}+\log (2)+\log (\sqrt{2}-1))}{(\log (e-1)-1)} \approx 1.70 \ldots
$$

and

$$
\max \curlywedge_{1}=(\sqrt{2}+\log (2)+\log (\sqrt{2}-1)) / \log (e-1) \approx 2.2 \ldots
$$

$$
\min \curlywedge_{2}=-(e-1)(-2+\sqrt{2}+\log (2)-\log (1+\sqrt{2})) \approx 1.329 \ldots
$$

and

$$
\max \curlywedge_{2}=\frac{((e-1)(\sqrt{2}+\log (2)-\log (1+\sqrt{2})))}{(e-2)} \approx 2.932 \ldots
$$


Proof State the convex univalent function $\varrho_{e}(z)=\frac{e^{z}-1}{z}$. It is clear that $\varrho(0)=1$ with a positive real part. Moreover, it satisfies the inequality

$$
\frac{e-1}{e} \leq \Re\left(\varrho_{e}(z)\right) \leq e-1, \quad z \in \mathbb{U} .
$$

By the conversation of Theorem 3.1, we indicate the following inequality:

$$
\frac{e-1}{e} \leq \Gamma_{\curlywedge}(-1) \leq \Gamma_{\curlywedge}(1) \leq e-1
$$

if $\curlywedge$ has the upper and lower bounds

$$
\min \curlywedge_{0}=\frac{(\sqrt{2}+\log (2)+\log (\sqrt{2}-1))}{(e-2)} \approx 1.706 \ldots
$$

and

$$
\max \curlywedge_{0}=-e(-2+\sqrt{2}+\log (2)-\log (1+\sqrt{(2)})) \approx 2.10399 \ldots
$$

This establishes the subordination inequalities

$$
\Gamma_{\curlywedge}(z) \prec \frac{e^{z}-1}{z} \Rightarrow P(z) \prec \frac{e^{z}-1}{z}, \quad z \in \mathbb{U} .
$$

Similarly, we have

$$
\min \curlywedge_{1}=\frac{(-2+\sqrt{2}+\log (2)+\log (\sqrt{2}-1))}{(\log (e-1)-1)} \approx 1.70 \ldots
$$

and

$$
\max \curlywedge_{1}=(\sqrt{2}+\log (2)+\log (\sqrt{2}-1)) / \log (e-1) \approx 2.2 \ldots
$$

This yields the subordination inequalities

$$
\Pi_{\curlywedge}(z) \prec \frac{e^{z}-1}{z} \Rightarrow P(z) \prec \frac{e^{z}-1}{z}, \quad z \in \mathbb{U} .
$$

Lastly, we get the upper and lower bounds

$$
\min \curlywedge_{2}=-(e-1)(-2+\sqrt{2}+\log (2)-\log (1+\sqrt{2})) \approx 1.329 \ldots
$$

and

$$
\max \curlywedge_{2}=\frac{((e-1)(\sqrt{2}+\log (2)-\log (1+\sqrt{2})))}{(e-2)} \approx 2.932 \ldots .
$$

This admits the subordination inequalities

$$
\Theta_{\curlywedge}(z) \prec \frac{e^{z}-1}{z} \Rightarrow P(z) \prec \frac{e^{z}-1}{z}, \quad z \in \mathbb{U} .
$$




\section{Majorization}

We progress to involve the formula

$$
\aleph_{\varphi}^{k}(z)=a_{k} \varphi^{k}(z)+a_{k-1} \varphi^{k-1}(z)+\cdots+a_{1} \varphi(z)+a_{0}
$$

for some $k$ to investigate the geometric solutions of Eq. (2.1). Dividing Eq. (2.1) by $\alpha_{1} \neq 0$, we receive

$$
\varphi^{\prime 3}(z)-\frac{\alpha_{2}}{\alpha_{1}} \varphi^{\prime 2}(z) \varphi(z)-\frac{\alpha_{3}}{\alpha_{1}} \varphi^{\prime}(z) \varphi^{2}(z)=-\frac{\aleph_{\varphi}^{k}(z)}{\alpha_{1}}, \quad z \in \mathbb{C} .
$$

We have the following result.

Theorem 4.1 Consider CADE (4.1) with $\alpha_{1}=-1$ and $a_{0}=1$. If $\varphi \in M(\rho)$ is a convex univalent function in $\mathbb{U}$ fulfilling the restriction of Theorem 3.1, then the communication values $a_{l}$ have the following construction:

$$
a_{1}=-\frac{1}{2}, \quad a_{2}=\frac{7}{12}, \quad a_{3}=-\frac{8}{12}, \quad a_{4}=\frac{74}{100}, \quad a_{5}=-\frac{79}{100} .
$$

Proof From Eq. (4.1) together with Theorem 3.1, we have $\aleph_{\varphi}^{k}(z) \prec \rho_{e}(z)$. Since $\varphi$ is convex univalent in $\cup$, then it indicates the extreme function construction

$$
\varphi(z)=\frac{z}{(1-z)}=z+z^{2}+\cdots .
$$

Thus, we obtain

$$
\begin{aligned}
\aleph_{\varphi}^{0}(z)= & 1 \\
\aleph_{\varphi}^{1}(z)= & 1+a_{1} z+a_{1} z^{2}+a_{1} z^{3}+a_{1} z^{4}+a_{1} z^{5}+O\left(z^{6}\right), \\
\aleph_{\varphi}^{2}(z)= & 1+a_{1} z+\left(a_{1}+a_{2}\right) z^{2}+\left(a_{1}+2 a_{2}\right) z^{3}+\left(a_{1}+3 a_{2}\right) z^{4}+\left(a_{1}+4 a_{2}\right) z^{5}+O\left(z^{6}\right), \\
\aleph_{\varphi}^{3}(z)= & 1+a_{1} z+\left(a_{1}+a_{2}\right) z^{2}+\left(a_{1}+2 a_{2}+a_{3}\right) z^{3}+\left(a_{1}+3\left(a_{2}+a_{3}\right)\right) z^{4} \\
& +\left(a_{1}+4 a_{2}+6 a_{3}\right) z^{5}+O\left(z^{6}\right), \\
\aleph_{\varphi}^{4}(z)= & 1+a_{1} z+\left(a_{1}+a_{2}\right) z^{2}+\left(a_{1}+2 a_{2}+a_{3}\right) z^{3}+\left(a_{1}+3 a_{2}+3 a_{3}+a_{4}\right) z^{4} \\
& +\left(a_{1}+4 a_{2}+6 a_{3}+4 a_{4}\right) z^{5}+O\left(z^{6}\right), \\
\aleph_{\varphi}^{5}(z)= & 1+a_{1} z+\left(a_{1}+a_{2}\right) z^{2}+\left(a_{1}+2 a_{2}+a_{3}\right) z^{3}+\left(a_{1}+3 a_{2}+3 a_{3}+a_{4}\right) z^{4} \\
& +\left(a_{1}+4 a_{2}+6 a_{3}+4 a_{4}+a_{5}\right) z^{5}+O\left(z^{6}\right), \\
\aleph_{\varphi}^{6}(z)= & 1+a_{1} z+\left(a_{1}+a_{2}\right) z^{2}+\left(a_{1}+2 a_{2}+a_{3}\right) z^{3}+\left(a_{1}+3 a_{2}+3 a_{3}+a_{4}\right) z^{4} \\
& +\left(a_{1}+4 a_{2}+6 a_{3}+4 a_{4}+a_{5}\right) z^{5}+O\left(z^{6}\right),
\end{aligned}
$$

Moreover, we attain that

$$
\rho_{e}(z)=\frac{z}{e^{z}-1}=\sum_{n=0}^{\infty} \frac{B_{n} z^{n}}{n !}
$$


where $B_{n}$ is the Bernoulli numbers having the inequality

$$
\begin{aligned}
& \left|B_{n}\right| \ll 4 \sqrt{\pi n}\left(\frac{n}{\pi e}\right)^{2 n}, \quad B_{2 n+1}=0, \\
& \left(B_{0}=1, B_{1}=-1 / 2, B_{2}=1 / 6, B_{4}=-1 / 30, B_{6}=1 / 42\right) .
\end{aligned}
$$

Comparing the coefficients of $\aleph_{\varphi}^{k}(z)$ and $\rho_{e}(z)$, we have

$$
\begin{aligned}
& a_{1}=\frac{B_{1}}{1 !}=-\frac{1}{2} \\
& a_{2}=-a_{1}+\frac{B_{2}}{2 !}=\frac{7}{12} \\
& a_{3}=-a_{1}-2 a_{2}+\frac{B_{3}}{3 !}=-\frac{8}{12} \\
& a_{4}=-a_{1}-3 a_{2}-3 a_{3}+\frac{B_{4}}{4 !}=\frac{74}{100}, \\
& a_{5}=-a_{1}-4 a_{2}-6 a_{3}-4 a_{4}+\frac{B_{5}}{5 !}=-\frac{79}{100} .
\end{aligned}
$$

The next result indicates the value of constant coefficients of $\aleph_{\varphi}^{k}$ when $\varphi$ is starlike in $\mathbb{U}$.

Theorem 4.2 Consider CADE (4.1) with $\alpha_{1}=-1$ and $a_{0}=1$. If $\varphi \in M(\rho)$ is a starlike function in $\mathbb{U}$ fulfilling the construction of Theorem 3.1, then the communication values $a_{t}$ have the construction

$$
a_{1}=-\frac{1}{2}, \quad a_{2}=\frac{13}{12}, \quad a_{3}=-\frac{28}{10}, \quad a_{4}=\frac{795}{100}, \quad a_{5}=-24
$$

Proof Plainly, from the conditions, we get $\aleph_{\varphi}^{k}(z) \prec \rho_{e}(z)$. Since $\varphi$ is starlike in $\cup$, then it has the extreme function formula

$$
\varphi(z)=\frac{z}{(1-z)^{2}}=z+2 z^{2}+\cdots
$$

Thus, we get

$$
\begin{aligned}
\aleph_{\varphi}^{0}(z)= & 1 \\
\aleph_{\varphi}^{1}(z)= & 1+a_{1} z+2 a_{1} z^{2}+3 a_{1} z^{3}+4 a_{1} z^{4}+5 a_{1} z^{5}+O\left(z^{6}\right) \\
\aleph_{\varphi}^{2}(z)= & 1+a_{1} z+\left(2 a_{1}+a_{2}\right) z^{2}+\left(3 a_{1}+4 a_{2}\right) z^{3}+\left(4 a_{1}+10 a_{2}\right) z^{4} \\
& +5\left(a_{1}+4 a_{2}\right) z^{5}+O\left(z^{6}\right) \\
\aleph_{\varphi}^{3}(z)= & 1+a_{1} z+\left(2 a_{1}+a_{2}\right) z^{2}+\left(3 a_{1}+4 a_{2}+a_{3}\right) z^{3}+\left(4 a_{1}+10 a_{2}+6 a_{3}\right) z^{4} \\
& +\left(5 a_{1}+20 a_{2}+21 a_{3}\right) z^{5}+O\left(z^{6}\right) \\
\aleph_{\varphi}^{4}(z)= & 1+a_{1} z+\left(2 a_{1}+a_{2}\right) z^{2}+\left(3 a_{1}+4 a_{2}+a_{3}\right) z^{3}+\left(4 a_{1}+10 a_{2}+6 a_{3}+a_{4}\right) z^{4} \\
& +\left(5 a_{1}+20 a_{2}+21 a_{3}+8 a_{4}\right) z^{5}+O\left(z^{6}\right) \\
\aleph_{\varphi}^{5}(z)= & 1+a_{1} z+\left(2 a_{1}+a_{2}\right) z^{2}+\left(3 a_{1}+4 a_{2}+a_{3}\right) z^{3}+\left(4 a_{1}+10 a_{2}+6 a_{3}+a_{4}\right) z^{4}
\end{aligned}
$$




$$
\begin{aligned}
& +\left(5 a_{1}+20 a_{2}+21 a_{3}+8 a_{4}+a_{5}\right) z^{5}+O\left(z^{6}\right), \\
\aleph_{\varphi}^{6}(z)= & 1+a_{1} z+\left(2 a_{1}+a_{2}\right) z^{2}+\left(3 a_{1}+4 a_{2}+a_{3}\right) z^{3}+\left(4 a_{1}+10 a_{2}+6 a_{3}+a_{4}\right) z^{4} \\
& +\left(5 a_{1}+20 a_{2}+21 a_{3}+8 a_{4}+a_{5}\right) z^{5}+O\left(z^{6}\right),
\end{aligned}
$$

Contrasting the connections of $\aleph_{\varphi}^{m}(z)$ and $\rho_{e}(z)$, we have

$$
\begin{aligned}
& a_{1}=\frac{B_{1}}{1 !}=-\frac{1}{2}, \\
& a_{2}=-2 a_{1}+\frac{B_{2}}{2 !}=\frac{13}{12}, \\
& a_{3}=-3 a_{1}-4 a_{2}+\frac{B_{3}}{3 !}=-\frac{28}{10}, \\
& a_{4}=-4 a_{1}-10 a_{2}-6 a_{3}+\frac{B_{4}}{4 !}=\frac{795}{100}, \\
& a_{5}=-5 a_{1}-20 a_{2}-21 a_{3}-8 a_{4}+\frac{B_{5}}{5 !}=-24 .
\end{aligned}
$$

\section{Remark 4.3}

- Note that Theorems 4.1 and 4.2 indicate that $\aleph_{\varphi}^{k}(z)$ accumulates at $k=5$, which yields the expansion formulas

$$
\aleph_{z /(1-z)}^{5}=1-\frac{z}{2}+\frac{z^{2}}{12}-\frac{z^{4}}{100}+O\left(z^{6}\right)
$$

and

$$
\aleph_{z /(1-z)^{2}}^{5}=1-\frac{z}{2}+\frac{8 z^{2}}{100}-\frac{2 z^{4}}{100}+O\left(z^{6}\right)
$$

- One can extend Theorems 4.1 and 4.2 in expressions of $\alpha$ for all estimates. In this suggestion, we get the constant connections $\mathbb{k}_{l}=\frac{a_{l}}{-\alpha_{1}}$ provided $\alpha \neq 0$

$$
\mathbb{k}_{1}=-\frac{1}{2}, \quad \mathbb{k}_{2}=\frac{7}{12}, \quad \mathbb{k}_{3}=-\frac{8}{12}, \quad \mathbb{k}_{4}=\frac{74}{100}, \quad \mathbb{k}_{5}=-\frac{79}{100}
$$

and

$$
\mathbb{k}_{1}=-\frac{1}{2}, \quad \mathbb{k}_{2}=\frac{13}{12}, \quad \mathbb{k}_{3}=-\frac{28}{10}, \quad \mathbb{k}_{4}=\frac{795}{100}, \quad \mathbb{k}_{5}=-24,
$$

respectively.

\section{Acknowledgements}

The authors would like to thank the editorial office for their advice. Also, the authors wish to express their profound gratitude to the anonymous referees for their careful reading of the manuscript and the very useful comments that have been implemented in the final version of the manuscript. 
Abbreviations

ABBDE, Algebraic Briot-Bouquet differential equations.

\section{Availability of data and materials}

Not applicable.

Ethics approval and consent to participate

Not applicable.

\section{Competing interests}

The authors declare that they have no competing interests.

\section{Consent for publication}

Not applicable.

\section{Authors' contributions}

All authors contributed equally and significantly in writing this article. All authors read and approved the final manuscript.

\section{Author details}

'Informetrics Research Group, Ton Duc Thang University, Ho Chi Minh City, Vietnam. ${ }^{2}$ Faculty of Mathematics \& Statistics, Ton Duc Thang University, Ho Chi Minh City, Vietnam. ${ }^{3}$ Department of Mathematics, Cankaya University, 06530 Balgat, Ankara, Turkey. ${ }^{4}$ Institute of Space Sciences, R76900 Magurele-Bucharest, Romania. ${ }^{5}$ Department of Medical Research, China Medical University, Taichung 40402, Taiwan.

\section{Publisher's Note}

Springer Nature remains neutral with regard to jurisdictional claims in published maps and institutional affiliations.

Received: 22 September 2020 Accepted: 23 November 2020 Published online: 01 December 2020

\section{References}

1. Ibrahim, R.W., Jahangiri, J.M., Cloud Computing Center: Conformable differential operator generalizes the Briot-Bouquet differential equation in a complex domain. AIMS Math. 4(6), 1582-1595 (2019)

2. Ibrahim, R.W., Darus, M.: On a class of analytic functions associated to a complex domain concerning q-differential-difference operator. Adv. Differ. Equ. 2019(1), 515 (2019)

3. Dreyfus, T., Lastra, A., Malek, S.: On the multiple-scale analysis for some linear partial q-difference and differentia equations with holomorphic coefficients. Adv. Differ. Equ. 2019(1), 326 (2019)

4. Ibrahim, R.W., Hadid, S.B., Momani, S.: Generalized Briot-Bouquet differential equation by a quantum difference operator in a complex domain. Int. J. Dyn. Control 8, 762-771 (2020)

5. Ibrahim, R.W., Elobaid, R.M., Obaiys, S.J.: Symmetric conformable fractional derivative of complex variables. Mathematics 8(3), 363 (2020)

6. Ibrahim, R.W., Elobaid, R.M., Obaiys, S.J.: Generalized Briot-Bouquet differential equation based on new differential operator with complex connections. Axioms 9(2), 42 (2020)

7. Shi, X., Liao, L., Zhang, S.: Explicit meromorphic solutions of a certain Briot-Bouquet differential equations. Chin. Ann. Math., Ser. B 41, 383-396 (2020)

8. Okada, Y., Schafke, R., Tahara, H.: A formal solvability of a coupling equation for PDEs of Briot-Bouquet type (microlocal analysis and asymptotic analysis). RIMS Kokyuroku 2101, 91-97 (2019)

9. Liao, L., Lu, X.: On solutions of a certain type of Briot-Bouquet equations. Complex Var. Elliptic Equ. 64(8), 1419-1436 (2019)

10. Saleeby, E.G.: On meromorphic solutions of first-order Briot-Bouquet type PDEs. J. Math. Anal. Appl. 482(1), 123517 (2020)

11. Acu, M., Oros, G.: Starlikeness condition for a new differential-integral operator. Mathematics 8(5), 694 (2020)

12. Antonino, J.A., Miller, S.S.: F-classes of univalent functions and F-differential subordinations. Comput. Methods Funct. Theory, 1-19 (2020)

13. Marshall, A.W., Olkin, I., Arnold, B.C.: Inequalities: Theory of Majorization and Its Applications, vol. 143. Academic Press, New York (1979)

14. Ibrahim, R.W.: A new approach of utility function based on fractional Gini aggregation operator for intelligent access web selection. SN Appl. Sci. 1(9), 1126 (2019)

15. Luptacik, M., Nezinsky, E.: Measuring income inequalities beyond the Gini coefficient. Cent. Eur. J. Oper. Res. 28, 561-578 (2020)

16. Wang, H.-Y., et al.: Comparison of Ferguson's $\delta$ and the Gini coefficient used for measuring the inequality of data related to health quality of life outcomes. Health Qual. Life Outcomes 18, 1-9 (2020)

17. Miller, S.S., Mocanu, P.T.: Differential Subordinations: Theory and Applications. CRC Press, Boca Raton (2000)

18. Gandhi, S., Kumar, S., Ravichandran, V.: First order differential subordinations for Caratheodory functions. Kyungpook Math. J. 58(2), 257-270 (2018) 